\title{
miR-149 suppresses human non-small cell lung cancer growth and metastasis by inhibiting the FOXM1/cyclin D1/MMP2 axis
}

\author{
LIJIANG ZHAO $^{1}$, LIJUAN LIU ${ }^{2}$, ZHENG DONG $^{1}$ and JIE XIONG ${ }^{1}$ \\ ${ }^{1}$ Department of Respiratory, Linyi Central Hospital, Linyi, Shandong 276400; \\ ${ }^{2}$ Department of Laboratory, People's Hospital of Rizhao, Rizhao, Shandong 276826, P.R. China
}

Received April 13, 2017; Accepted June 27, 2017

DOI: $10.3892 /$ or.2017.6047

\begin{abstract}
Valid evidence has demonstrated that microRNAs have critical functions in cancer genesis and tumor progression. In the present study, aberrant expression of microRNA-149 (miR-149) was confirmed in non-small cell lung cancer (NSCLC) tissues. Low expression of miR-149 was associated with malignant clinical features and poor survival. Gain- and loss-of-function experiments demonstrated that miR-149 inhibited NSCLC tumor growth and metastasis in vitro and in vivo. Furthermore, oncogenic transcription factor FOXM1 was confirmed as a direct target of miR-149 in NSCLC. Cyclin D1 and MMP2 served as downstream targets of FOXM1 and were also inhibited by miR-149. In summary, the present study indicated that downregulation of miR-149 in NSCLC predicted poor clinical outcomes. miR-149 suppresses tumor growth and metastasis in NSCLC by inhibiting the FOXM1/cyclin D1/MMP2 signaling pathway.
\end{abstract}

\section{Introduction}

Non-small cell lung cancer (NSCLC) is a common pathological type of lung cancers (1). NSCLC exerts rapid growth and aggressive features during its progression. Even though marked improvement in the diagnosis and treatment of NSCLC has been achieved, the prognosis of NSCLC patients particularly for those in advanced stages is still poor (2). Thus, it is imperative to investigate new biomarkers and treatment regimens for NSCLC.

MicroRNAs (miRNAs) are a group of small, non-protein coding, endogenous and single-stranded RNAs (3). miRNAs have been confirmed to play vital roles in many biological

Correspondence to: Dr Lijiang Zhao, Department of Respiratory, Linyi Central Hospital, Linyi, Shandong 276400, P.R. China E-mail: lchzhaolijiang@126.com

Key words: miR-149, growth, metastasis, non-small cell lung cancer, FOXM1 processes including cell growth (4), metastasis (5) and angiogenesis (6) by negatively regulating target mRNAs to either translation or degradation.

Recently, microRNA-149 (miR-149) was identified as a tumor inhibitor in human cancers. In hepatocellular carcinoma, low expression of miR-149 was found to be correlated with larger tumor mass size, capsular and vascular invasion, lymph node metastasis, high histological grade, advanced tumor-node-metastasis (TNM) stage and poor prognosis (7). In vitro, miR-149 inhibited cell proliferation, migration and invasion by targeting PPM1F (8). In gastric cancer, miR-149 was upregulated by a chemical compound named $18 \beta$-glycyrrhetinic acid (GRA) and inhibited the progression of gastric tumors by targeting the Wnt-1 signaling pathway (9). However, the expression and functions of miR-149 in NSCLC are still unclear.

In the present study, we investigated the expression and biological function of miR-149 in NSCLC progression. Downregulation of miR-149 was confirmed to be correlated with large tumor size, metastasis and poor prognosis in NSCLC patients. miR-149 suppressed NSCLC tumor growth and metastasis by targeting oncogenic transcription factor FOXM1 by gain- and loss-of-function experiments.

\section{Materials and methods}

Clinical samples and cell lines. Forty-five paired non-small cell lung cancer and matched tumor-adjacent tissues were collected at Linyi Central Hospital (Linyi, China). The patients included 31 males and 14 females who received surgical resection between March 2011 and January 2013. The age range was between 37 and 69 years. 293T cells, normal lung epithelium BEAS-2B and 4 NSCLC cell lines (A549, H1299, H1975 and HCC827) were purchased from the American Type Culture Collection (ATCC; Manassas, VA, USA) and cultured in Dulbecco's modified Eagle's medium (DMEM; Invitrogen, Waltham, MA, USA) supplemented with $10 \%$ fetal bovine serum (FBS; Gibco, Grand Island, NY, USA), $1 \%$ penicillin and $1 \%$ streptomycin.

Real-time quantitative reverse transcription-PCR ( $q R T-P C R)$. Total RNA from tissues and cells was isolated by RNeasy 
Plus Micro and Mini kits (cat no. 74034; Qiagen, Venlo, The Netherlands) according to the manufacturer's instructions. One-Step Perfect Real-Time RT-qPCR (SYBR-Green I) kit (Takara Biotechnology Co., Ltd., Dalian, China) was used to detected the expression levels of miR-149 and FOXM1 mRNA. miR-149 and RNU6 Bulge-Loop ${ }^{\mathrm{TM}}$ primers were purchased from RiboBio Co., Ltd. (Guangzhou, China). FOXM1 and GAPDH primers were synthesized by Sangon Co., Ltd. (Shanghai, China). Data were analyzed using the $2^{-\Delta \Delta \mathrm{Ct}}$ method.

Cell transfection. miR-149 and negative control (miR-NC) lentivirus particles were purchased from GeneCopoeia (Guangzhou, PR China). miR-NC or miR-149 and package plasmids were co-transfected into $293 \mathrm{~T}$ cells using Lipofectamine $^{\mathrm{TM}} 2000$ (Invitrogen) reagent according to the manufacturer's instructions. The supernatant including the lentivirus was collected twice at 48 and $72 \mathrm{~h}$ after transfection. Cells $\left(15 \times 10^{4} /\right.$ well $)$ were seeded into a 6 -well plate before the infection day. For lentivirus infection, $1 \mathrm{ml}$ lentivirus supernatant (miR-NC or miR-149) combined with $2 \mathrm{ml}$ growth medium and Polybrene $(5 \mu \mathrm{g} / \mathrm{ml})$ were added into one well. Puromycin was used to select the successfully infected cells. The effect of transfection was determined by qRT-PCR.

Anti-BrdU cell proliferation assay. For cell proliferation detection, we used an anti-BrdU assay according to the manufacturer's recommendation (BD Biosciences, San Jose, CA, USA). Cells were cultured into a 24-well plate. BrdU solution $(0.1 \mathrm{mg} / \mathrm{ml})$ was added to each well. Cells were incubated at $37^{\circ} \mathrm{C}$ for $4 \mathrm{~h}$. The incorporated BrdU was stained with Alexa Fluor ${ }^{\circledR} 488$ anti-BrdU monoclonal antibody (BD Biosciences). Cell nuclei were stained with DAPI. Positive stained cells were observed and counted under a fluorescence microscope.

Cell apoptosis assay. To evaluate the apoptosis of NSCLC cells, the flow cytometric assay was performed to evaluate the percentage of apoptotic cells. In brief, cells were harvested and re-suspended in phosphate-buffered saline (PBS) solution, and then stained with Annexin V-FITC/PI detection kit (556547; BD Biosciences) and subjected to FACS analysis.

Wound healing assay. Cells were seeded into a 6-well plate until achieving $90 \%$ confluency. A pippette tip (100 $\mu \mathrm{l})$ was used to make a wound in the middle of the well. Remnant cells were washed away using PBS. Basal DMEM was used to culture cells for $48 \mathrm{~h}$. The healing of the wound was observed under an inverted microscope.

Transwell assay. The upper face of $8-\mu \mathrm{m}$ pore Transwell inserts (Nalge Nunc International, Penfield, NY, USA) was coated with Matrigel (BD Biosciences, Franklin Lakes, NJ, USA) for the invasion assay. In brief, cells suspended with basal DMEM were seeded onto the upper chamber. DMEM (750 $\mu \mathrm{l}$ ) with $10 \%$ FBS was added into the lower chambers, and. $24 \mathrm{~h}$ later, cells that had invaded the membranes and stayed on the lower surface were stained with crystal violet. The invaded cell number was counted under a microscope.
Table I. Clinical significance of miR-149 expression in NSCLC $(\mathrm{N}=45)$.

\begin{tabular}{|c|c|c|c|c|}
\hline \multirow[b]{2}{*}{$\begin{array}{l}\text { Clinical } \\
\text { characteristics }\end{array}$} & \multicolumn{2}{|c|}{$\begin{array}{c}\text { miR-149 } \\
\text { expression } \\
\text { level }\end{array}$} & \multirow[b]{2}{*}{$\chi^{2}$} & \multirow[b]{2}{*}{ P-value } \\
\hline & $\begin{array}{l}\text { High } \\
n=20\end{array}$ & $\begin{array}{l}\text { Low } \\
n=25\end{array}$ & & \\
\hline \multicolumn{5}{|l|}{ Sex } \\
\hline Male & 15 & 16 & 0.627 & 0.525 \\
\hline Female & 5 & 9 & & \\
\hline \multicolumn{5}{|l|}{ Age (years) } \\
\hline$<50$ & 9 & 17 & 2.409 & 0.142 \\
\hline$\geq 50$ & 11 & 8 & & \\
\hline \multicolumn{5}{|l|}{ Smoking } \\
\hline Yes & 8 & 15 & 1.779 & 0.236 \\
\hline No & 12 & 10 & & \\
\hline \multicolumn{5}{|c|}{ Tumor size $(\mathrm{cm})$} \\
\hline$\geq 3$ & 5 & 18 & 9.823 & $0.003^{\mathrm{a}}$ \\
\hline$<3$ & 15 & 7 & & \\
\hline \multicolumn{5}{|l|}{ Metastasis } \\
\hline Yes & 3 & 14 & 7.946 & $0.006^{\mathrm{a}}$ \\
\hline No & 17 & 11 & & \\
\hline \multicolumn{5}{|l|}{ TNM stage } \\
\hline $\mathrm{I}+\mathrm{II}$ & 13 & 8 & 4.862 & $0.038^{\mathrm{a}}$ \\
\hline $\mathrm{III}+\mathrm{IV}$ & 7 & 17 & & \\
\hline
\end{tabular}

${ }^{\mathrm{a}} \mathrm{P}<0.05$. TNM, tumor-node-metastasis; miR-149 microRNA-149; NSCLC, non-small cell lung cancer.

In vivo experiments. Genetic modified and wild-type tumor cells were subcutaneously injected into nude mice to investigate the functions of miR-149 on tumor growth or injected into the left pleural cavity of the mice for tumor metastasis. Mice were sacrificed after 4 weeks. Final tumor volumes (V) were calculated based on the following formula: $\mathrm{V}=$ (length $\mathrm{x}$ width $\left.{ }^{2}\right) / 2$. Mouse livers were harvested to make paraffin-embedded tissue sections for H\&E staining.

Western blotting. Total cell proteins were extracted with RIPA buffer, separated by $10 \%$ SDS-PAGE gel and transferred onto a nitrocellulose membrane (Invitrogen). The membranes were incubated with the following primary rabbit anti-human antibodies purchased from Cell Signaling Technology Inc. (CST; Danvers, MA, USA) at $4^{\circ} \mathrm{C}$ overnight: FOXM1 (5436; dilution at 1:1,000), cyclin D1 (2978; dilution at 1:1,000), MMP2 (87809; dilution at 1:1,000) and GAPDH $(5174$; dilution at 1:1,000). Then, the membranes were incubated with HRP-linked goat anti-rabbit antibodies (7074; dilution at 1:5,000; CST), and the signals were detected using the Bio-Rad Gel imaging system (Bio-Rad, Hercules, CA, USA).

Luciferase reporter assay. Stable miR-149 or miR-NC infected cells were grown in a 24 -well plate until achieving 
A

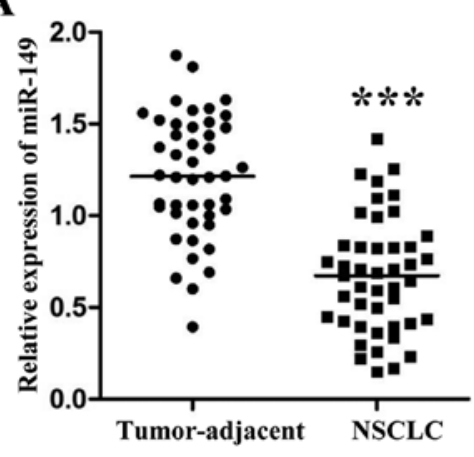

Tissue type

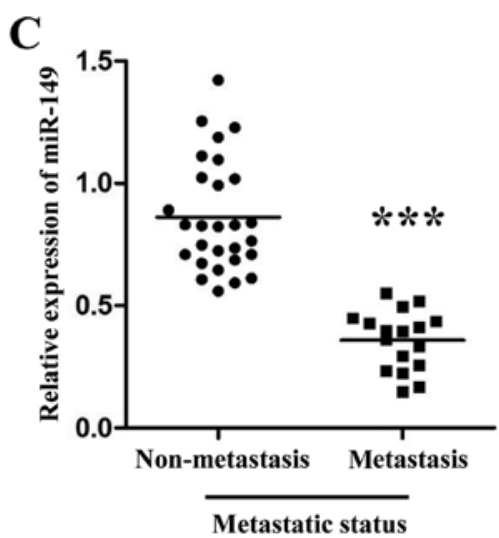

B

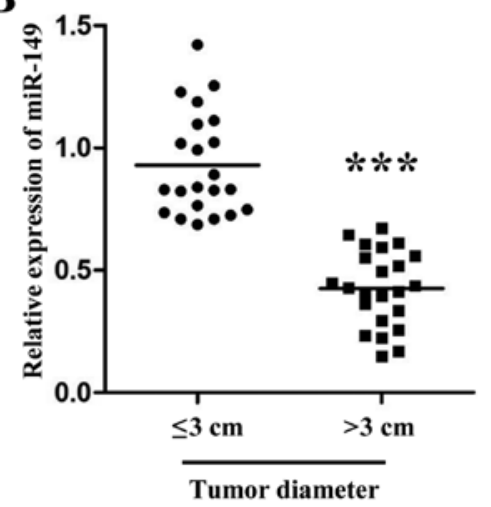

D

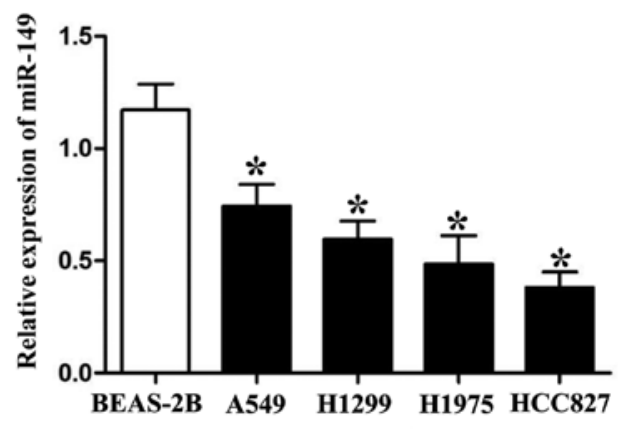

Figure 1. The expression levels of miR-149 in NSCLC tissues and cell lines. (A) miR-149 was downregulated in NSCLC tissues. Patients with (B) large tumor diameter and (C) metastasis expressed lower miR-149 levels. (D The expression levels of miR-149 were downregulated in the NSCLC cell lines; ${ }^{* * *} \mathrm{P}<0.001$, ${ }^{*} \mathrm{P}<0.05$.

$50 \%$ confluency. Cells were transfected with $50 \mathrm{ng}$ of wildtype (WT) or mutant (MT) 3'-untranslated region (3'-UTR) vectors of FOXM1 (RiboBio Co., Ltd.) in a 24-well plate using Lipofectamine $^{\mathrm{TM}} 2000$ (Invitrogen) reagent according to the manufacturer's instructions for $48 \mathrm{~h}$. The hLuc fluorescence intensity and hRluc fluorescence intensity were detected by the Dual-Luciferase Reporter Assay System (Promega, Madison, WI, USA) following the manufacturer's instructions. The ratio of Rluc/Luc was used to measure the effects of combination between miR-149 and 3'-UTR of FOXM1.

Statistical analysis. All quantitative data are expressed as mean \pm SD. SPSS v21.0 software (IBM, Armonk, NY, USA) was used to perform statistical analysis. The Pearson's Chi-square test was used for enumeration data and the Student's t-test was performed for measurement of data in the present study. Overall survival and progression-free survival analysis were performed using the Kaplan-Meier method for plotting and the log-rank test for comparison. $\mathrm{P}<0.05$ was indicative of a statistically significant difference.

\section{Results}

miR-149 is downregulated in NSCLC tissues and cell lines. qRT-PCR was used to detect the expression levels of miR-149 in cancer tissues and different NSCLC cell lines. As shown in Fig. 1A, the expression levels of miR-149 were downregulated in the NSCLC tissues (Fig. 1A; P<0.001). Furthermore, we also divided these 45 patients into different subgroups according to their clinical features. As shown in Fig. 1B and C, PCR results revealed that patients with large tumor size and metastasis had lower miR-149 expression levels than those who did not have these malignant features $(\mathrm{P}<0.001$, respectively). Next, compared with normal lung epithelium cells, we also found that miR-149 was downregulated in 4 NSCLC cell lines (A549, H1299, H1975 and HCC827; Fig. 1D; P<0.05, respectively).

Clinical significance of miR-149 in NSCLC. To further investigate the clinical significance of miR-149, we used the mean expression level of miR-149 ( $\mathrm{x}=0.672)$ as a cut-off value to divide 45 NSCLC tissues into 2 groups: miR-149 high expression group $(n=20)$ and miR-149 low expression group $(n=25)$. As shown in Table I, low expression of miR-149 was associated with large tumor size $(\mathrm{P}=0.003)$, tumor metastasis $(\mathrm{P}=0.006)$ and advanced TNM stage $(\mathrm{P}=0.038)$. We also compared the differences in the 3-year survival rates between these 2 groups. As shown in Fig. 2, patients with low levels of miR-149 had a poor 3-year overall survival rate (Fig. $2 \mathrm{~A}$; $\mathrm{P}<0.05$ ) and diseasefree survival rate (Fig. $2 \mathrm{~B} ; \mathrm{P}<0.05$ ). These findings indicated that miR-149 could serve as a biomarker for NSCLC patients.

miR-149 inhibits the proliferation and induces apoptosis of NSCLC cells. Following the analysis of expression levels of miR-149 in the 4 NSCLC cell lines, we used miR-149 lentivirus to overexpress its expression level in HCC827 cells and the anti-miR-149 lentivirus to knock down its expression level 
A

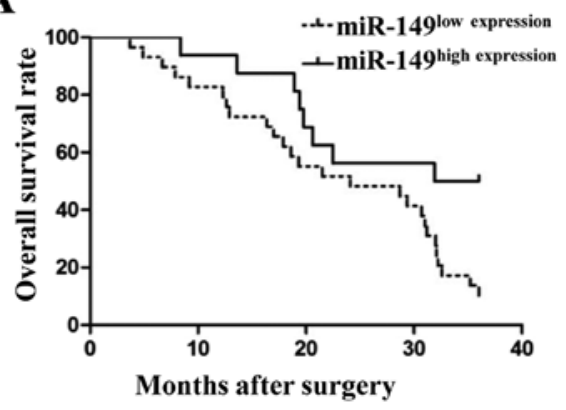

B

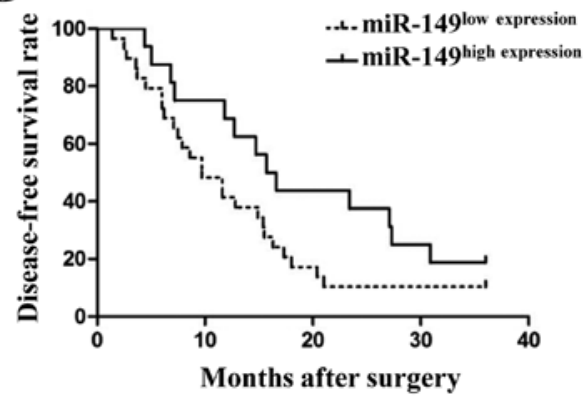

Figure 2. Low expression of miR-149 is associated with poor 3-year prognosis. Patients were divided into 2 groups according to the expression levels of miR-149. Patients in low miR-149 expression group showed lower (A) overall survival and (B) disease-free survival rates than patients in the high miR-149 expression group.

$\mathbf{A}$
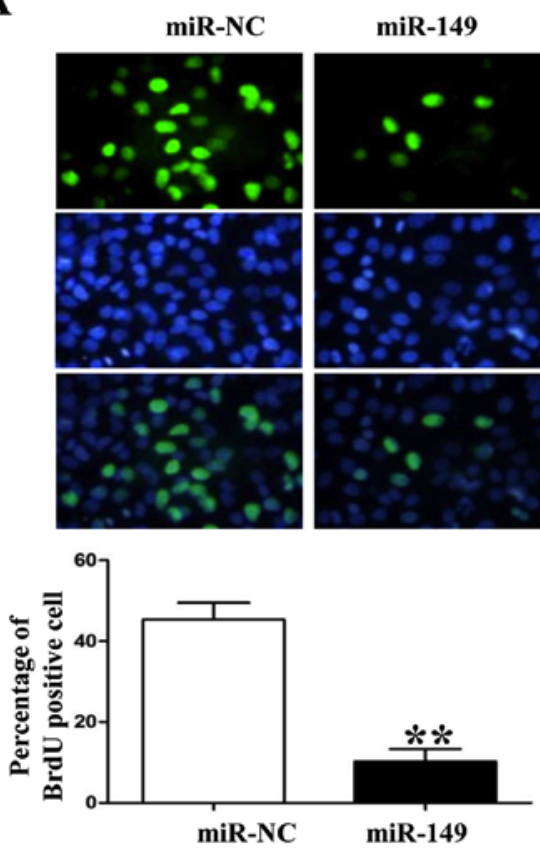

C
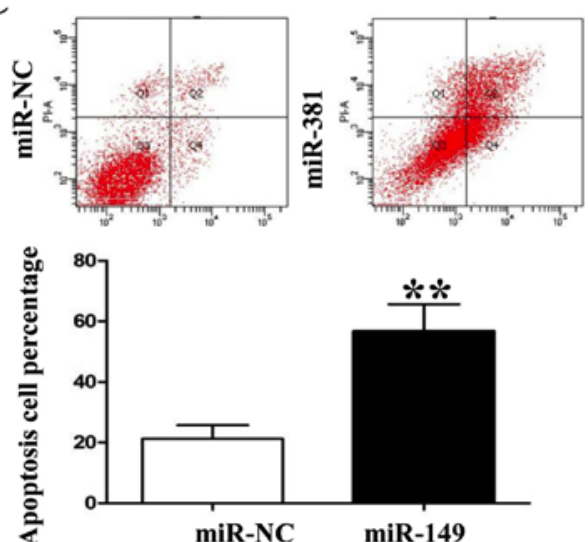

B
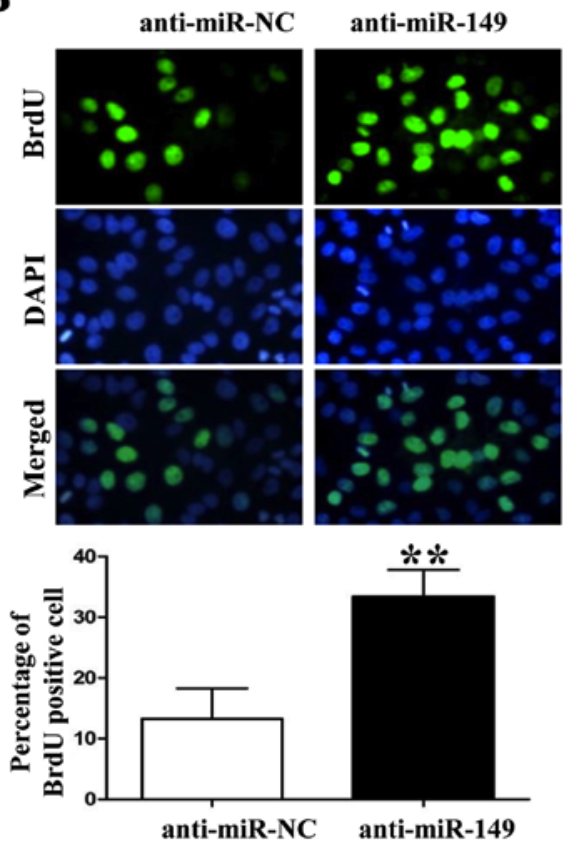

D
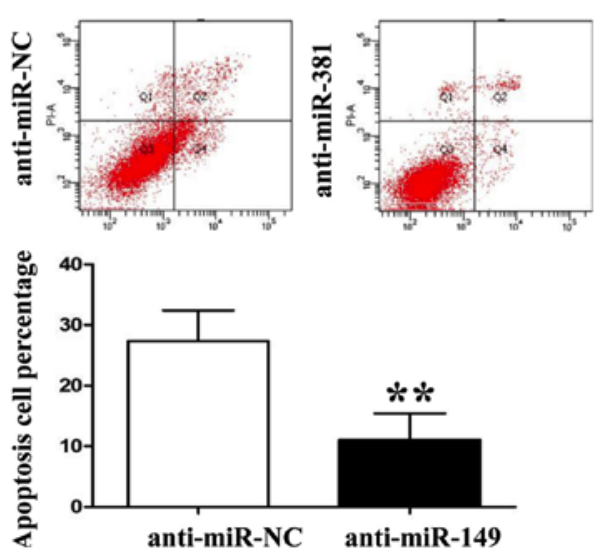

Figure 3. miR-149 inhibits proliferation and induces apoptosis of NSCLC cells in vitro. Cell proliferation and apoptosis were measured by anti-BrdU assay and flow cytometry, respectively. miR-149 overexpression (A) inhibited proliferation and (C) induced apoptosis of HCC827 cells. Inhibition of miR-149 (B) increased the proliferation viability and (D) decreased the apoptosis of A549 cells; ${ }^{* *} \mathrm{P}<0.01$.

in A549 cells $(\mathrm{P}<0.05$, data not shown). Anti-BrdU assay showed that miR-149 overexpression significantly decreased the number of BrdU-positive staining HCC827 cells (Fig. 3A; $\mathrm{P}<0.01)$. However, miR-149 knockdown induced A549 
A
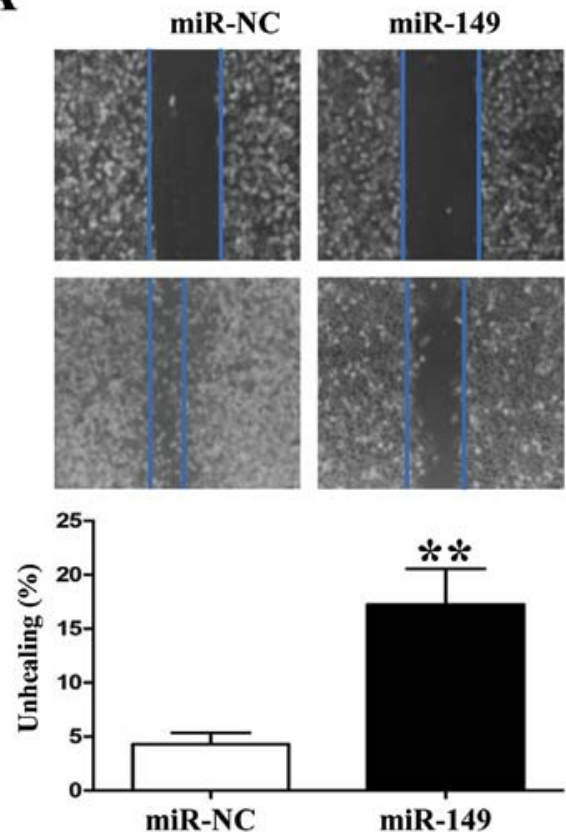

C
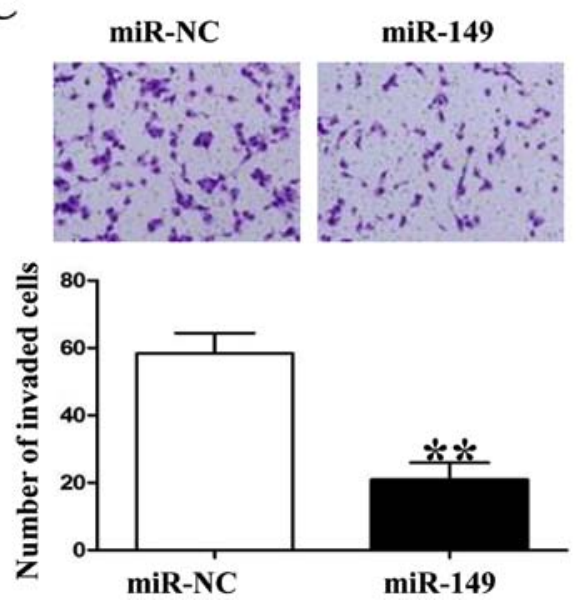

B
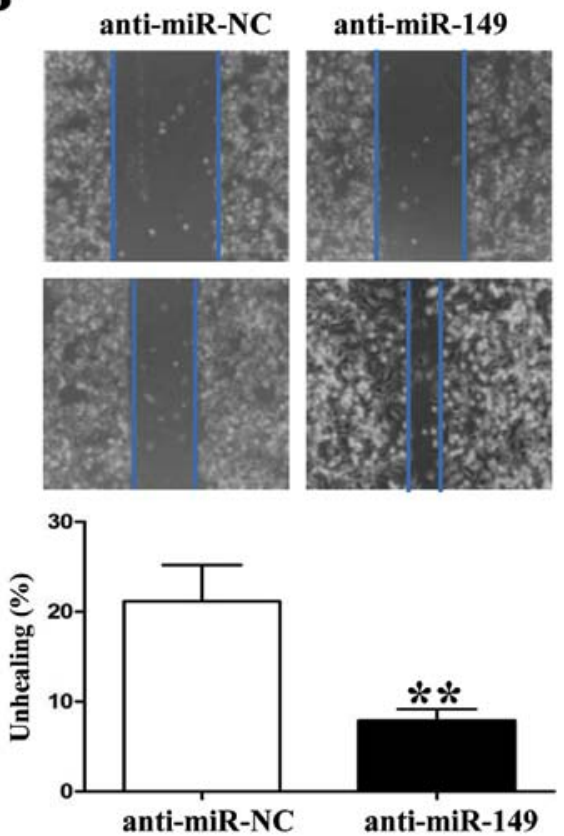

D
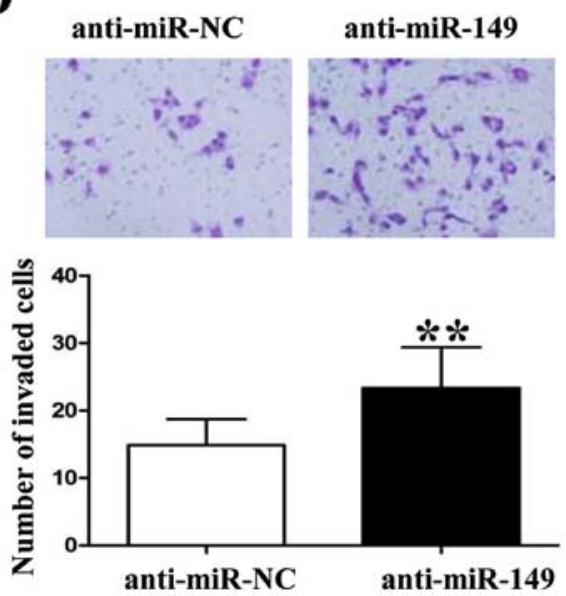

Figure 4. miR-149 inhibits migration and invasion of NSCLC cells in vitro. Cell migration and invasion were measured by wound-healing and Transwell assays, respectively. Upregulation of miR-149 inhibited (A) migration and (C) invasion of HCC827 cells. Downregulation of miR-149 promoted (B) migration and (D) invasion of A549 cells; ${ }^{* *} \mathrm{P}<0.01$.

cell proliferation based on the BrdU immunofluorescence results (Fig. 3B; $\mathrm{P}<0.01$ ). In contrast, flow cytometric assay showed that miR-149 overexpression increased the percentage of apoptotic HCC827 cells whereas miR-149 knockdown decreased A549 cell apoptosis (Fig. 3C and D; P<0.01, respectively).

miR-149 inhibits the migration and invasion of NSCLC cells. Since miR-149 was found to be associated with tumor metastasis in clinical samples, we also used wound healing and Transwell assays to demonstrate that upregulation of miR-149 impaired the migration and invasion of HCC827 cells (Fig. 4A and C; $\mathrm{P}<0.01$, respectively). In contrast, downregulation of miR-149 in A549 cells enhanced their migration and invasion markedly (Fig. 4B and D; P<0.01, respectively). In summary, these data suggest that miR-149 attenuates the metastatic ability of NSCLC cells.
miR-149 inhibits NSCLC tumor growth and metastasis in vivo. When we demonstrated that miR-149 inhibited NSCLC growth and metastasis in vitro, we also established a tumor subcutaneous growth model and liver metastasis model to further confirm the anticancer roles of miR-149 in vivo. As shown in Fig. 5A and B, forced expression of miR-149 in NSCLC cells inhibited the tumor growth in nude mice $(\mathrm{P}<0.05$, respectively). Subsequently, overexpression of miR-149 significantly reduced the liver metastasis of NSCLC cells (Fig. 5C and D; $\mathrm{P}<0.05$, respectively). These data suggest that miR-149 plays a key role in the growth and metastasis of NSCLC cells in vitro and in vivo.

miR-149 exerts its anticancer functions by inhibiting FOXM1 in NSCLC cells. FOXM1 was predicted as a potential downstream target of miR-149 according to bioinformatic analysis by online database screening (TargetScan: 
A

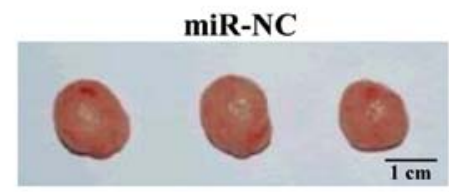

miR-149
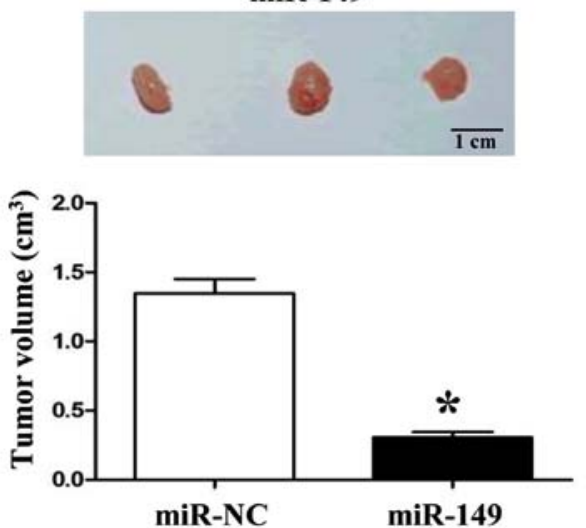

C
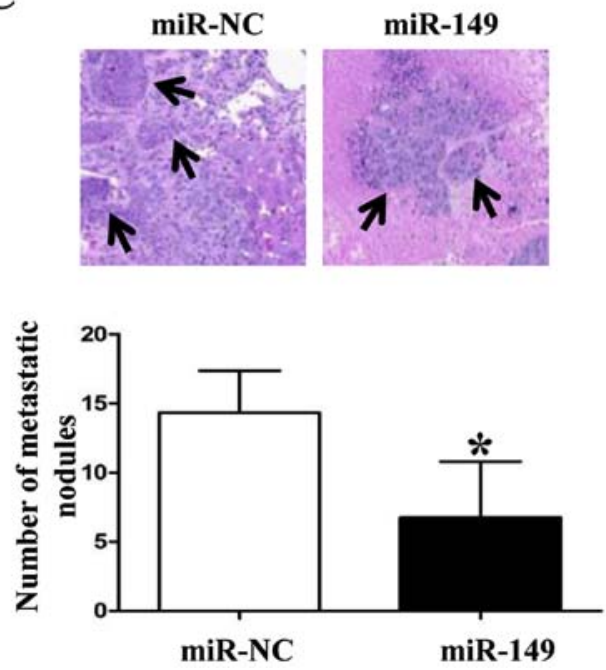

B
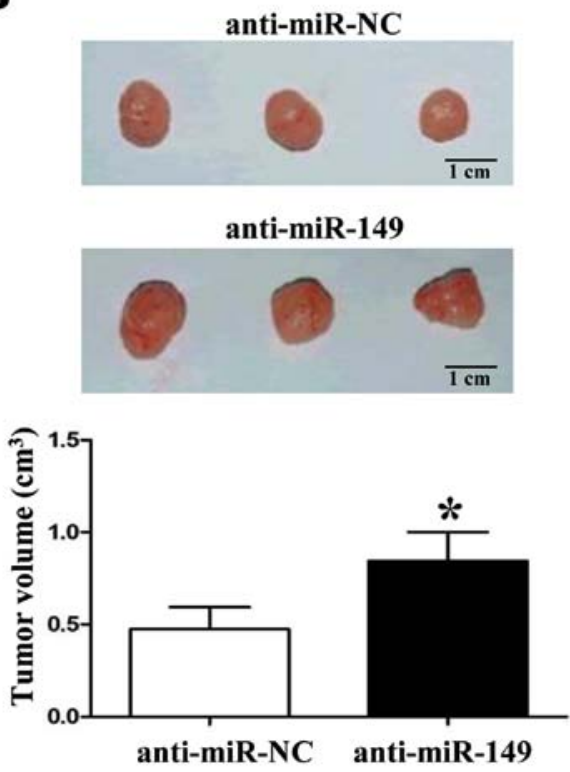

D
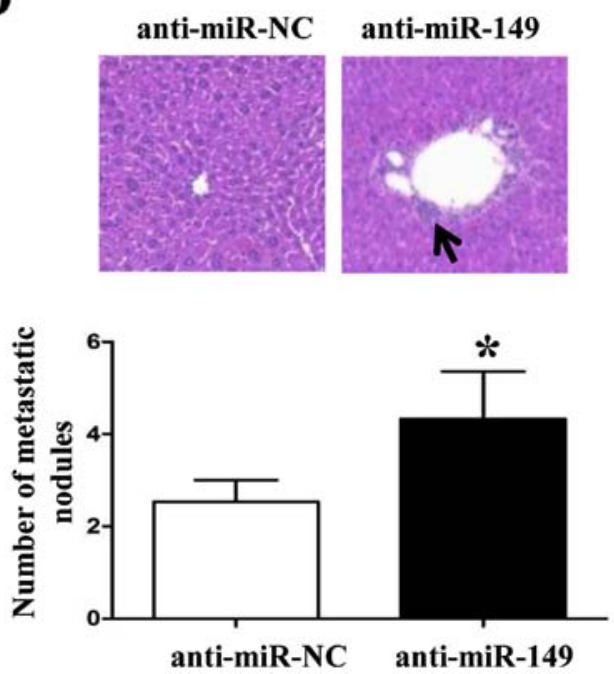

Figure 5. miR-149 inhibits NSCLC growth and metastasis in vivo. Overexpression of miR-149 in HCC827 cells inhibited (A) tumor volume and (C) liver metastasis; * $\mathrm{P}<0.05$. miR-149 knockdown in A549 cells promoted (B) tumor growth and (D) liver metastasis. Liver metastatic clusters are labeled by black arrows in $(\mathrm{C}$ and $\mathrm{D}) ;{ }^{*} \mathrm{P}<0.05$.

http://www.targetscan.org/ and miRanda: http://www. microrna.org/). To confirm the relationship between miR-149 and FOXM1, first, we detected the expression of FOXM1 in miR-194-overexpressing HCC827 and miR-194-knockdown A549 cells. Both the mRNA and protein expression levels of FOXM1 were downregulated when we overexpressed miR-149 in the HCC827 cells (Fig. 6A; $\mathrm{P}<0.01$ and $\mathrm{P}<0.05$, respectively), whereas FOXM1 was upregulated when miR-149 was inhibited in the A549 cells (Fig. 6B; $\mathrm{P}<0.01$ and $\mathrm{P}<0.05$, respectively). Secondly, we used a Dual-Luciferase ${ }^{\circledR}$ Reporter Assay System to confirm that only the luciferase activity of wild-type FOXM1 3'-UTR was downregulated by miR-149 (Fig. 6C; P<0.01). Finally, we detected the mRNA levels of FOXM1 in NSCLC tissues by qRT-PCR and demonstrated that the mRNA expression of FOXM1 was lower in the miR-149 high expression group than that noted in the miR-149 low expression group (Fig. 6D; $\mathrm{P}<0.05$ ). In the present study, we found that the expression levels of cyclin D1 and MMP2 were also negatively regulated by miR-149 in the NSCLC cells (Fig. 7A and B; P<0.01). Cyclin D1 and MMP2 are not targets of miR-149, but they have been demonstrated to be regulated by FOXM1 $(10,11)$. Thus, these results may partly explain the anticancer mechanisms of miR-149 in NSCLC.

\section{Discussion}

Tumor growth and metastasis are two vital risk factors for the poor prognosis of NSCLC patients (12). Numerous studies have demonstrated that microRNAs (miRNAs) are critical players in the genesis and progression of human cancers, particularly in NSCLC. miR-502e was found to serve as a tumor suppressor and inhibit NSCLC cell growth, invasion and migration by targeting Zbtb7a partly depending on the Wnt signaling pathway (13). However, miR-30a reduced radiation-induced 
A
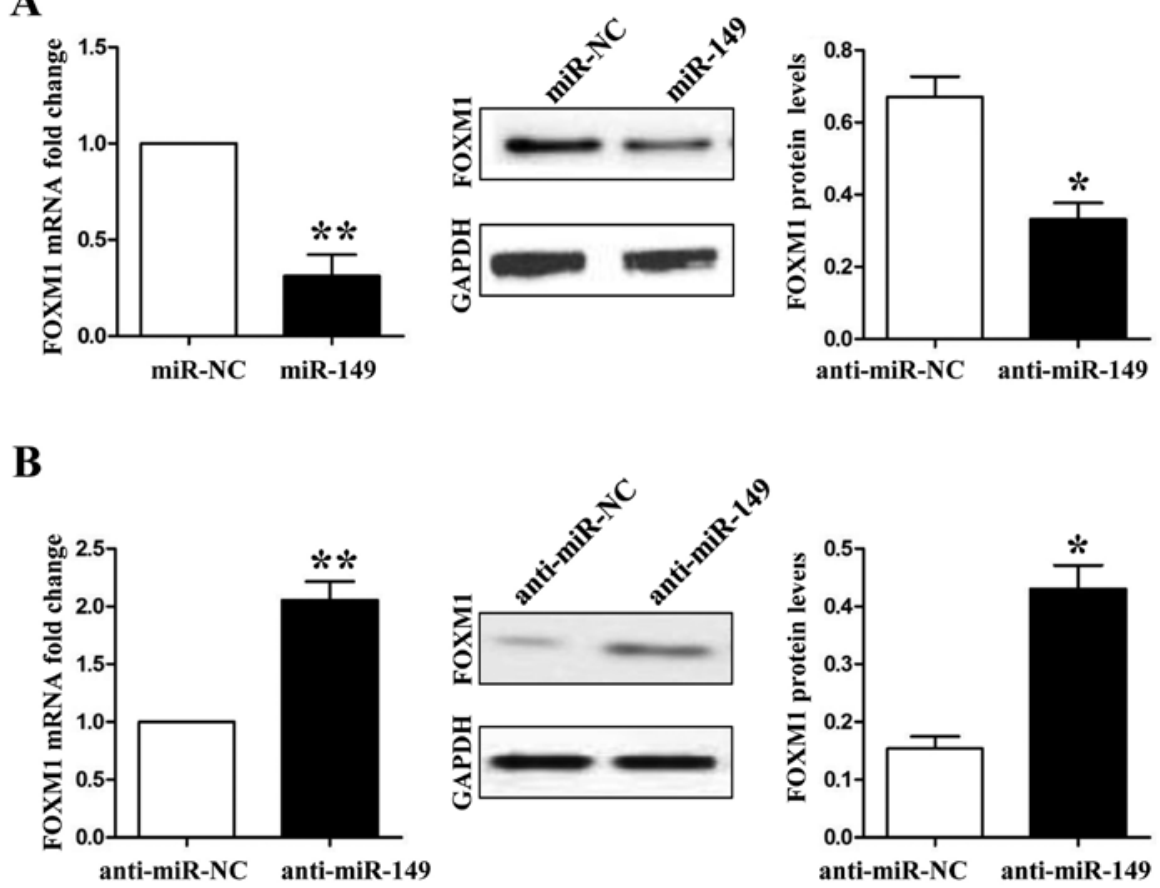

C

D
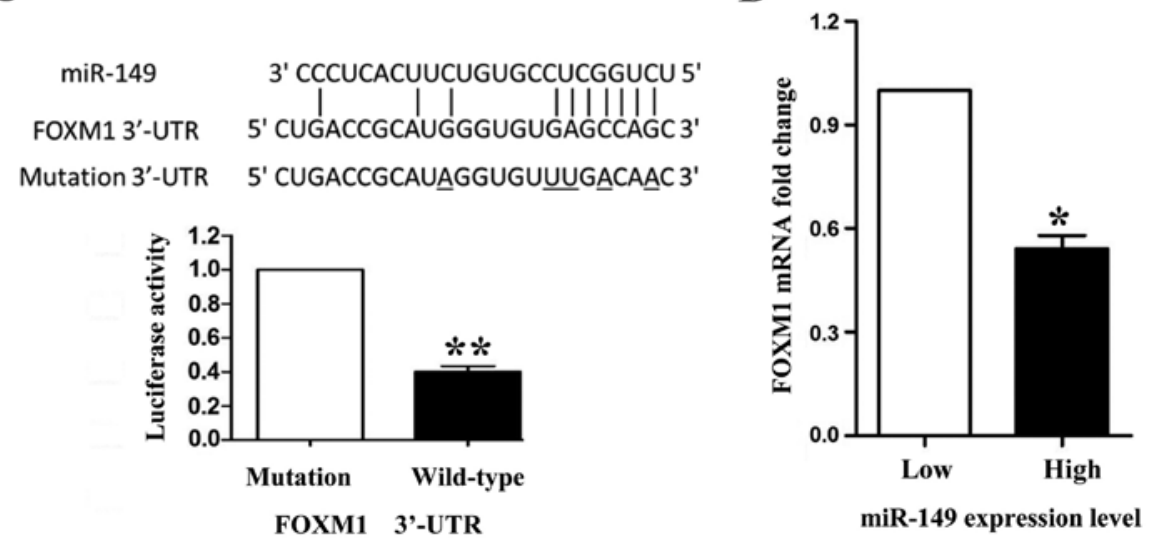

Figure 6. FOXM1 is a direct target of miR-149 in NSCLS cells. (A) Overexpression of miR-149 significantly decreased FOXM1 mRNA and protein expression levels. (B) miR-149 knockdown markedly increased FOXM1 mRNA and protein expression levels. (C) The wild-type and mutant type sequences of FOXM1 3'-UTR for miR-149 were designed. miR-149 only decreased the luciferase activity that carried wild-type but not mutant type 3'-UTR of FOXM1. (D) Patients in the miR-149 high expression group had low FOXM1 mRNA expression levels; $\mathrm{P}<0.05,{ }^{* *} \mathrm{P}<0.01$.

G2/M cell cycle arrest and may also affect radiation-induced apoptosis by targeting ATF1 (14). This accumulating evidence shows that miRNAs are promising biomarkers and therapeutic targets in NSCLC.

In the present study, we revealed that miR-149 was significantly decreased in NSCLC cancer tissues and cell lines. Clinical data showed that low expression of miR-149 was associated with large tumor size, tumor metastasis and advanced TNM stage. In addition, low expression levels of miR-149 predicted a reduced 3-year overall and disease-free survival rates of NSCLC patients. Taken together, these data indicated that miR-149 may play a critical role in the development of NSCLC.

Functionally, we used gain- and loss-of-function experiments to confirm that miR-149 inhibited NSCLC cell proliferation, migration and invasion in vitro and it also inhibited subcutaneous xenograft growth and liver metastasis in vivo. These results were consistent with the expression levels of miR-149 in the clinical samples.

Forkhead box protein M1 (FOXM1) is a member of the FOX family of transcription factors. In NSCLC, FOXM1 has been demonstrated as a tumor inducer due to its function in tumor proliferation (15), invasion (16) and chemoresistance (17). Moreover, FOXM1 was found to be a target of several miRNAs in NSCLC such as miR-134 (18) and miR-509 (19). In the present study, we found that FOXM1 may be a target of miR-149. To confirm their relationship, we used PCR, western blot and luciferase reporter assays to demonstrate that miR-149 inhibited FOXM1 mRNA and protein expression levels by binding to its 3'-UTR in NSCLC cells. Moreover, patients with low expression levels of miR-149 exerted high FOXM1 mRNA levels. As a classical oncogenic transcription factor in NSCLC, 
$\mathbf{A}$
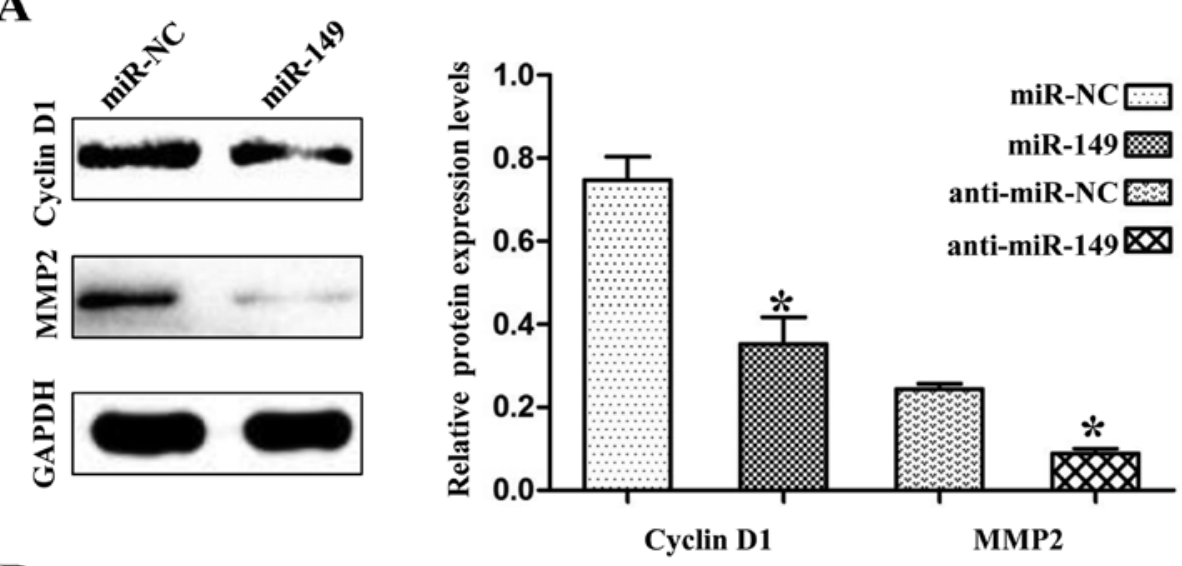

$\mathbf{B}$
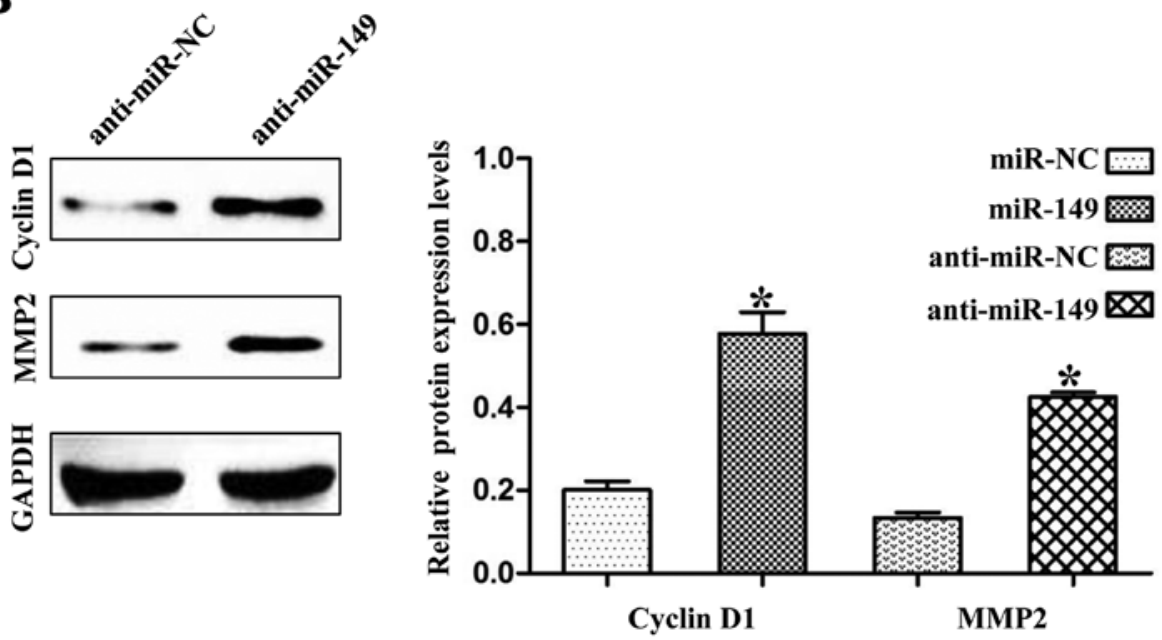

Figure 7. miR-149 inhibits cyclin D1 and MMP2 expression in NSCLC cells. (A) miR-149 decreased the expression of cyclin D1 and MMP2 in HCC827 cells. (B) Downregulation of miR-149 increased the expression of cyclin D1 and MMP2 in A549 cells; * $\mathrm{P}<0.05$.

FOXM1 could enhance the transcription of many oncogenes including cyclin D1 and MMP2 in lung cancer $(11,20,21)$. In addition, we also found that miR-149 decreased the expression levels of cyclin D1 and MMP2 in NSCLC cells.

In conclusion, we demonstrated that miR-491 was downregulated in NSCLC tissues and cell lines, and its reduced expression was associated with malignant clinical features. miR-149 can inhibit NSCLC growth and metastatic behaviors by inhibiting FOXM1. These results suggest that miR-149 is a potential tumor-suppressor in NSCLC.

\section{References}

1. Gadgeel SM: Personalized therapy of non-small cell lung cancer (NSCLC). Adv Exp Med Biol 890: 203-222, 2016.

2. Califano R, Romanidou O, Mountzios G, Landi L, Cappuzzo F and Blackhall F: Management of NSCLC disease progression after first-line EGFR tyrosine kinase inhibitors: What are the issues and potential therapies? Drugs 76: 831-840, 2016.

3. Ameis D, Khoshgoo N, Iwasiow BM, Snarr P and Keijzer R: MicroRNAs in lung development and Disease. Paediatr Respir Rev 22: 38-43, 2017.

4. Singh S, Zheng Y, Jagadeeswaran G, Ebron JS, Sikand K, Gupta S, Sunker R and Shukla GC: Deep sequencing of small RNA libraries from human prostate epithelial and stromal cells reveal distinct pattern of microRNAs primarily predicted to target growth factors. Cancer Lett 371: 262-273, 2016.

5. Zoni E and van der Pluijm G: The role of microRNAs in bone metastasis. J Bone Oncol 5: 104-108, 2016.
6. Masotti A, Miller MR, Celluzzi A, Rose L, Micciulla F, Hadoke PW, Bellucci S and Caporali A: Regulation of angiogenesis through the efficient delivery of microRNAs into endothelial cells using polyamine-coated carbon nanotubes. Nanomedicine 12: 1511-1522, 2016.

7. Lin L, Zhang YD, Chen ZY, Chen Y and Ren CP: The clinicopathological significance of miR-149 and PARP-2 in hepatocellular carcinoma and their roles in chemo/radiotherapy. Tumour Biol 37: 12339-12346, 2016.

8. Luo G, Chao YL, Tang B, Li BS, Xiao YF, Xie R, Wang SM, Wu YY, Dong H, Liu XD, et al: miR-149 represses metastasis of hepatocellular carcinoma by targeting actin-regulatory proteins PPM1F. Oncotarget 6: 37808-37823, 2015.

9. Cao D, Jia Z, You L, Wu Y, Hou Z, Suo Y, Zhang H, Wen S, Tsukamoto T, Oshima M, et al: $18 \beta$-glycyrrhetinic acid suppresses gastric cancer by activation of miR-149-3p-Wnt-1 signaling. Oncotarget 7: 71960-71973, 2016.

10. Gao F, Bian F, Ma X, Kalinichenko VV and Das SK: Control of regional decidualization in implantation: Role of FoxM1 downstream of Hoxa10 and cyclin D3. Sci Rep 5: 13863, 2015.

11. Chen PM, Wu TC, Shieh SH, Wu YH, Li MC, Sheu GT, Cheng YW, Chen CY and Lee H: MnSOD promotes tumor invasion via upregulation of FoxM1-MMP2 axis and related with poor survival and relapse in lung adenocarcinomas. Mol Cancer Res 11: 261-271, 2013.

12. Chen YY, Huang TW, Tsai WC, Lin LF, Cheng JB, Chang H and Lee SC: Risk factors of postoperative recurrences in patients with clinical stage I NSCLC. World J Surg Oncol 12: 10,2014

13. Zhijun Z and Jingkang H: MicroRNA-520e suppresses nonsmall-cell lung cancer cell growth by targeting Zbtb7a-mediated Wnt signaling pathway. Biochem Biophys Res Commun 486: 49-56, 2017. 
14. Guo Y, Sun W, Gong T, Chai Y, Wang J, Hui B, Li Y, Song L and Gao Y: miR-30a radiosensitizes non-small cell lung cancer by targeting ATF1 that is involved in the phosphorylation of ATM. Oncol Rep 37: 1980-1988, 2017.

15. Kim IM, Ackerson T, Ramakrishna S, Tretiakova M, Wang IC, Kalin TV, Major ml, Gusarova GA, Yoder HM, Costa RH, et al: The Forkhead Box $\mathrm{m} 1$ transcription factor stimulates the proliferation of tumor cells during development of lung cancer. Cancer Res 66: 2153-2161, 2006.

16. Kong FF, Qu ZQ, Yuan HH, Wang JY, Zhao M, Guo YH, Shi J, Gong XD, Zhu YL, Liu F, et al: Overexpression of FOXM1 is associated with EMT and is a predictor of poor prognosis in non-small cell lung cancer. Oncol Rep 31: 2660-2668, 2014.

17. Wang K, Zhu X, Zhang K, Zhu L and Zhou F: FoxM1 inhibition enhances chemosensitivity of docetaxel-resistant A549 cells to docetaxel via activation of $\mathrm{JNK} /$ mitochondrial pathway. Acta Biochim Biophys Sin 48: 804-809, 2016.
18. Li J, Wang Y, Luo J, Fu Z, Ying J, Yu Y and Yu W: miR-134 inhibits epithelial to mesenchymal transition by targeting FOXM1 in non-small cell lung cancer cells. FEBS Lett 586: 3761-3765, 2012.

19. Ma N, Zhang W, Qiao C, Luo H, Zhang X, Liu D, Zang S, Zhang L and Bai J: The tumor suppressive role of miRNA-509-5p by targeting FOXM1 in non-small cell lung cancer. Cell Physiol Biochem 38: 1435-1446, 2016.

20. Liu Y, Hock JM, Van Beneden RJ and Li X: Aberrant overexpression of FOXM1 transcription factor plays a critical role in lung carcinogenesis induced by low doses of arsenic. Mol Carcinog 53: 380-391, 2014.

21. Balli D, Zhang Y, Snyder J, Kalinichenko VV and Kalin TV: Endothelial cell-specific deletion of transcription factor FoxM1 increases urethane-induced lung carcinogenesis. Cancer Res 71: 40-50, 2011. 